\title{
Analysis of Mixed Finite Element Methods for the Stokes Problem: A Unified Approach
}

\author{
By Rolf Stenberg
}

\begin{abstract}
We develop a method for the analysis of mixed finite element methods for the Stokes problem in the velocity-pressure formulation. A technical "macroelement condition", which is sufficient for the classical Babuška-Brezzi inequality to be valid, is introduced. Using this condition,we are able to verify the stability, and optimal order of convergence, of several known mixed finite element methods.
\end{abstract}

1. Introduction. The mixed finite element method, based on the velocity-pressure formulation, is being increasingly used for the numerical solution of the Navier-Stokes equations. In this paper we will discuss the mixed finite element method for the linear Stokes problem. Under suitable existence and uniqueness conditions the results can be extended to the nonlinear Navier-Stokes equations; cf. [11].

The analysis of mixed methods for the Stokes problem can be based on the general theory of saddle point problems developed by Babuška [1] and Brezzi [5]. The main difficulty in the analysis is the verification of the basic stability inequality, usually referred to as the Babuška-Brezzi inequality. In Crouzeix and Raviart [9] a rather general analysis technique, for triangular finite element subspaces, is developed. Recently a variant of the classical stability inequality has been introduced by Bercovier and Pironneau [4] for the analysis of methods where the pressure is approximated by continuous functions. It should also be mentioned that some methods, which are used in practice (cf. [13], [21]), do not fulfill the classical Babuska-Brezzi stability inequality. It is, however, possible to analyze these methods using certain weaker stability inequalities, cf. Johnson and Pitkäranta [15] and Pitkäranta [19], where error estimates for some methods have been derived.

In this paper we develop a general method for the analysis of mixed finite element methods for the Stokes problem. We introduce a technical "macroelement condition" which is easy to check and sufficient for the stability inequality (in its classical form) to be valid. A similar condition is used in [20] in the analysis of mixed methods for two-dimensional elasticity equations. Our method of analysis seems to both generalize and, above all, simplify the previous methods.

In order to avoid unnecessary technical details we have restricted ourselves to a two-dimensional, polygonal domain and to straight-sided triangular, or quadrilateral, elements. The method can easily be generalized to more general situations. In some of the examples we also treat general isoparametric elements.

Received February 8, 1983.

1980 Mathematics Subject Classification. Primary 65N30. 
The plan of the paper is as follows: In Section 2 we state the problem and its finite element discretization and give some preliminary results. The next section is devoted to the stability inequality. We introduce the macroelement condition and show how it implies the stability inequality. In Section 4 we apply our method of analysis to four mixed methods.

2. Preliminaries. Let $\Omega$ be a polygonal domain in $\mathbf{R}^{2}$ with boundary $\Gamma$. We consider the stationary Stokes problem: Find functions $u=\left(u_{1}, u_{2}\right)$ and $p$ defined on $\Omega$ such that

$$
\begin{array}{ll}
-\nu \Delta u+\nabla p=f & \text { in } \Omega, \\
\operatorname{div} u=0 & \text { in } \Omega, \\
u=0 & \text { on } \Gamma,
\end{array}
$$

where $u$ is the fluid velocity, $p$ is the pressure, $f$ is the body force and $\nu>0$ is the kinematic viscosity.

We denote by $|\cdot|_{s . T}$ and $\|\cdot\|_{s . T}$, respectively, the seminorm and norm of the Sobolev space $\left[H^{s}(T)\right]^{\alpha}$, where $s$ and $\alpha$ are integers. For noninteger $s, s \geqslant 0$, $\left[H^{s}(T)\right]^{\alpha}$ and $\|\cdot\|_{s . T}$ are defined as usual by interpolation. $H_{0}^{\prime}(T)$ denotes the subspace of $H^{1}(T)$ of functions vanishing on $\partial T$. We will also use the space

$$
L_{0}^{2}(T)=\left\{p \in L^{2}(T) \mid \int_{T} p d x=0\right\} .
$$

By $(\cdot, \cdot)_{T}$ we denote the inner product in $\left[L^{2}(T)\right]^{\alpha}$, where $\alpha$ is an integer. The subscript $T$ is omitted if $T=\Omega$.

Throughout the paper, $C$ and $C$, will stand for a positive constant, possibly different at different occurrences, which is independent of the mesh parameter $h$, but may depend on $\Omega, \nu$ and some other parameters introduced in the text.

Using the above notations, (2.1) allows the following weak formulation: Find $u \in\left[H_{0}^{1}(\Omega)\right]^{2}$ and $p \in L_{0}^{2}(\Omega)$ such that

$$
\begin{gathered}
\nu(\nabla u, \nabla v)-(\operatorname{div} v, p)=(f, v) \quad \forall v \in\left[H_{0}^{1}(\Omega)\right]^{2}, \\
(\operatorname{div} u, \mu)=0 \quad \forall \mu \in L_{0}^{2}(\Omega) .
\end{gathered}
$$

In the finite element discretization of $(2.2)$ we introduce the finite-dimensional subspaces $V_{h} \subset\left[H_{0}^{1}(\Omega)\right]^{2}$ and $P_{h} \subset L_{0}^{2}(\Omega)$ and formulate the approximate problem as: Find $u_{h} \in V_{h}$ and $p_{h} \in P_{h}$ such that

$$
\begin{gathered}
\nu\left(\nabla u_{h}, \nabla v\right)-\left(\operatorname{div} v, p_{h}\right)=(f, v) \quad \forall v \in V_{h}, \\
\left(\operatorname{div} u_{h}, \mu\right)=0 \quad \forall \mu \in P_{h} .
\end{gathered}
$$

In order to define the finite element spaces we introduce a partitioning $e_{h}$ of $\bar{\Omega}$ into subdomains which are assumed to be either triangles or convex quadrilaterals whose diameters are bounded by $h$. Given an element $K \in \mathcal{e}_{h}$, we denote by $h_{K}$ the diameter of $K$, by $\rho_{K}$ the maximum diameter of all circles contained in $K$ and by $\theta_{i K}$, $1 \leqslant i \leqslant 4$, the angles of $K$ if $K$ is a quadrilateral. We suppose that the family $e_{h}$ is regular in the sense that there exist two constants $\sigma>1$ and $0<\gamma<1$ independent of $h$ such that

$$
h_{K} \leqslant \sigma \rho_{K} \quad \forall K \in e_{h},
$$

$$
\left|\cos \theta_{i K}\right| \leqslant \gamma, \quad 1 \leqslant i \leqslant 4 \text {, for all quadrilaterals } K \in \mathcal{e}_{h} \text {. }
$$


Now, for each integer $m \geqslant 0$ we denote by $P_{m}(K)$ the space of polynomials of degree $\leqslant m$ on $K$ and by $Q_{m}(K)$ the space

$$
Q_{m}(K)=\left\{p=\hat{p} \circ F_{K}^{-1} \mid \hat{p} \in Q_{m}(\hat{K})\right\},
$$

where $\hat{K}$ is the unit sphere, $Q_{m}(\hat{K})$ is the space of polynomials of the form

$$
\hat{p}(x)=\sum_{0 \leqslant i, j \leqslant m} a_{i j} x_{1}^{i} x_{2}^{j}, \quad a_{i j} \in \mathbf{R},
$$

and $F_{K}$ is a bilinear transformation which maps $\hat{K}$ onto $K$. Setting

$$
R_{m}(K)= \begin{cases}P_{m}(K) & \text { if } K \text { is a triangle, } \\ Q_{m}(K) & \text { if } K \text { is a quadrilateral, }\end{cases}
$$

the space $V_{h}$ is defined as

$$
V_{h}=\left\{v=\left(v_{1}, v_{2}\right) \in\left[H_{0}^{1}(\Omega)\right]^{2} \mid v_{i \mid K} \in R_{k}(K), i=1,2, \forall K \in \bigodot_{h}\right\}
$$

Since the pressure does not need to be continuous, we have various possibilities of choosing $P_{h}$. A continuous pressure is obtained by defining

$$
P_{h}=\left\{p \in L_{0}^{2}(\Omega) \cap C(\bar{\Omega}) \mid p_{\mid K} \in R_{l}(K) \forall K \in \bigodot_{h}\right\} .
$$

We will also consider the following alternatives for a discontinuous pressure

$$
\begin{aligned}
& P_{h}=\left\{p \in L_{0}^{2}(\Omega) \mid P_{\mid K} \in R_{l}(K) \forall K \in \mathcal{C}_{h}\right\}, \\
& P_{h}=\left\{p \in L_{0}^{2}(\Omega) \mid p_{\mid K} \in P_{l}(K) \forall K \in \mathcal{C}_{h}\right\} .
\end{aligned}
$$

Remark. (2.9c) defines $p_{\mid K} \in P_{l}(K)$ also for quadrilaterals. This can occasionally be a good choice; cf. Example 4 in Section 4.

The spaces $V_{h}$ and $P_{h}$ have the following well-known (cf. [6], [7]) approximation properties.

LEMMA 2.1. If $u \in\left[H^{r}(\Omega) \cap H_{0}^{1}(\Omega)\right]^{2}, r \geqslant 1$, then there exist $\tilde{u} \in V_{h}$ such that $\|u-\tilde{u}\|_{1} \leqslant C h^{q_{1}-1}\|u\|_{q_{1}}$, where $q_{1}=\min \{r, k+1\}$.

LEMMA 2.2. If $p \in H^{s}(\Omega) \cap L_{0}^{2}(\Omega), s \geqslant 0$, then there exist $\tilde{p} \in P_{h}$ such that $\|p-\tilde{p}\|_{0} \leqslant C h^{q_{2}}\|p\|_{q_{2}}$, where $q_{2}=\min \{s, l+1\}$.

The Babuska-Brezzi stability condition [5], [11] for the approximate problem (2.3) is satisfied if there is a constant $C>0$ such that

$$
\sup _{\substack{v \in V_{h} \\ v \neq 0}} \frac{(\operatorname{div} v, p)}{|v|_{1}} \geqslant C\|p\|_{0} \quad \forall p \in P_{h} .
$$

This condition is fundamental for the analysis of the mixed method since it, together with Lemmas 2.1 and 2.2, implies the following error estimates (cf. [11]).

THEOREM 2.1. Suppose that the solution of (2.1) satisfies $u \in\left[H^{r}(\Omega)\right]^{2}, r \geqslant 1$, and $p \in H^{s}(\Omega), s \geqslant 0$, and let $\left(u_{h}, p_{h}\right)$ be the solution of (2.3). Then if the condition (2.10) is satisfied, we have the error estimate

$$
\left|u-u_{h}\right|_{1}+\left\|p-p_{h}\right\|_{0} \leqslant C\left(h^{q_{1}-1}\|u\|_{q_{1}}+h^{q_{2}}\|p\|_{q_{2}}\right)
$$


where $q_{1}=\min \{r, k+1\}$ and $q_{2}=\min \{s, l+1\}$. Moreover, if the region $\Omega$ is convex, we have the additional estimate

$$
\left\|u-u_{h}\right\|_{0} \leqslant C\left(h^{q_{1}}\|u\|_{q_{1}}+h^{q_{2}+1}\|p\|_{q_{2}}\right) .
$$

In the next section we will show how the stability condition (2.10) can be verified in practice.

3. The Stability Inequality. Let us start by introducing some additional notation. By a macroelement we mean the union of one or more neighboring triangles or quadrilaterals satisfying the regularity assumptions (2.4) and (2.5). A macroelement $M$ is said to be equivalent to a reference macroelement $\hat{M}$ if there is a mapping $F_{M}: \hat{M} \rightarrow M$ satisfying the conditions:

(i) $F_{M}$ is continuous and one-to-one.

(ii) $F_{M}(\hat{M})=M$.

(iii) If $\hat{M}=\cup_{j=1}^{m} \hat{K}_{j}$, where $\hat{K}_{j}, j=1,2, \ldots, m$, are the triangles or quadrilaterals in $\hat{M}$, then $K_{j}=F_{M}\left(\hat{K}_{j}\right), j=1,2, \ldots, m$, are the triangles or quadrilaterals in $M$.

(iv) $F_{M_{1} \hat{\kappa},}=F_{K} \circ F_{\hat{K}}^{-1}, j=1,2, \ldots, m$, where $F_{\hat{K}}$, and $F_{K}$ are the affine or bilinear mappings from the reference triangle (with vertices $(0,0),(0,1)$ and $(1,0))$ or unit square onto $\hat{K}_{j}$ and $K_{j}$, respectiveiy.

The family of macroelements equivalent with $\hat{M}$ will be denoted by $\mathcal{E}_{\hat{M}}$.

For a macroelement $M$ we define the space $V_{0, M}$ as

$$
V_{0, M}=\left\{v \in\left[H_{0}^{1}(M)\right]^{2} \mid v_{i \mid K} \in R_{k}(K), i=1,2, \forall K \subset M\right\} .
$$

Depending on which of the alternatives $(2.9 \mathrm{abc})$ is chosen to define $P_{h}$, we define the space $P_{M}$ respectively as

$$
\begin{aligned}
& P_{M}=\left\{p \in L^{2}(M) \cap C(\bar{M}) \mid p_{\mid K} \in R_{l}(K) \forall K \subset M\right\}, \\
& P_{M}=\left\{p \in L^{2}(M) \mid p_{\mid K} \in R_{l}(K) \forall K \subset M\right\}
\end{aligned}
$$

or

$$
P_{M}=\left\{p \in L^{2}(M) \mid p_{\mid K} \in P_{l}(K) \forall K \subset M\right\}
$$

We will further define

$$
P_{0, M}=P_{M} \cap L_{0}^{2}(M)
$$

and

$$
N_{M}=\left\{p \in P_{M} \mid(\operatorname{div} v, p)_{M}=0 \forall v \in V_{0, M}\right\}
$$

Let us now prove the following 
LeMma 3.1. Let $\mathscr{E}_{\hat{M}}$ be a class of equivalent macroelements. Suppose that for every $M \in \mathbb{E}_{\hat{M}}$, the space $N_{M}$ is one-dimensional, consisting of functions that are constant on $M$. Then there is a positive constant $\beta_{\hat{M}}=\beta(\hat{M}, \sigma, \gamma)$ such that the condition

$$
\sup _{\substack{v \in V_{0, M} \\ v \neq 0}} \frac{(\operatorname{div} v, p)_{M}}{|v|_{1, M}} \geqslant \beta_{\hat{M}}\|p\|_{0, M} \quad \forall p \in P_{0, M},
$$

holds for every $M \in \mathcal{E}_{\hat{M}}$.

Proof. Consider a fixed $M \in \mathcal{E}_{\hat{M}}$. Define the constant $\beta_{M}$ as

$$
\beta_{M}=\inf _{\substack{p \in P_{0, M} \\\|p\|_{0, M}=1}} \sup _{\substack{v \in V_{0, M} \\|v|_{1, M}=1}}(\operatorname{div} v, p)_{M}
$$

Since $N_{M}$ consists of functions that are constant on $M$, and $P_{0, M}$ and $V_{0, M}$ are finite dimensional, it follows that $\beta_{M}>0$.

Let us now prove that there is a constant $\beta_{\hat{M}}$ such that $\beta_{M} \geqslant \beta_{\hat{M}}>0$ for every $M \in \mathcal{E}^{(i)}$.

Let $\hat{x}^{1}, \hat{x}^{2}, \ldots, \hat{x}^{d}$ be the vertices of the triangles or quadrilaterals in $\hat{M}$. Every $M \in \mathcal{E}_{\hat{M}}$ is now uniquely defined by its vertices $x^{i}=F_{M}\left(\hat{x}^{i}\right), i=1,2, \ldots, d$, and so we may write $\beta_{M}=\beta\left(x^{1}, x^{2}, \ldots, x^{d}\right)$. We will now consider the vertices as a point $X=\left(x^{1}, x^{2}, \ldots, x^{d}\right)$ in $\mathbf{R}^{2 d}$, and $\beta_{M}=\beta(X)$ as a function of $X$. Let $h_{M}=$ $\max _{K \subset M}\left\{h_{K}\right\}$. We may assume that $h_{M}=1$ and that $x^{1}$ coincides with the origin in $\mathbf{R}^{2}$, since the general case can be handled by a scaling argument using the mapping $G(x)=h_{M}^{-1}\left(x-x^{1}\right)$. Since $x^{1}$ is chosen as the origin, every vertex $x^{1}, x^{2}, \ldots, x^{d}$ lies within a given distance from the origin. Further, every $K \subset M$ has a diameter less than or equal to unity and satisfies the regularity assumptions (2.4) and (2.5). This means that the point $X$ belongs to a compact set, denoted by $D$, in $\mathbf{R}^{2 d}$. It can now easily be proved that the function $\beta$ is continuous, and since $\beta(X)>0$ for every $X \in D$, we conclude that there is a constant $\beta_{\hat{M}}>0$ such that $\beta(X) \geqslant \beta_{\hat{M}}$ for every $X \in D$. We have thus proved the condition

$$
\inf _{\substack{p \in P_{0, M} \\\|p\|_{0, M}=1}} \sup _{\substack{v \in V_{0, M} \\ \mid v \|_{1, M}=1}}(\operatorname{div} v, p)_{M} \geqslant \beta_{\hat{M}}>0 \quad \forall M \in \mathcal{E}_{\hat{M}},
$$

which is equivalent to (3.5).

We are now ready to introduce a "macroelement condition" which is sufficient for the stability inequality (2.10) to be valid. Let us assume that there is a fixed set of classes $\mathcal{E}_{\hat{\mathcal{M}}}, i=1, \ldots, n, n \geqslant 1$, such that

For each $M \in \mathcal{E}_{\hat{M}_{i}}, i=1, \ldots, n$, the space $N_{M}$ is one-dimensional, consisting of functions that are constant on $M$.

Let us further assume that for each $h$ the triangles or quadrilaterals in $\bigodot_{h}$ can be grouped together to form macroelements such that the so obtained macroelement partitioning $\mathfrak{R}_{h}$ of $\bar{\Omega}$ satisfies the following condition:

$$
\begin{aligned}
& \text { Each } M \in \Re_{h} \text { belongs to some of the classes } \varepsilon_{\hat{M}_{i}}, \\
& i=1,2, \ldots, n .
\end{aligned}
$$


In the case when linear and bilinear elements are used for the velocities we will need one additional condition:

If $k=1$ in (2.8) and $T$ is the common part of the boundaries of two macroelements in : $T_{h}$, then $T$ is connected and contains at least two edges of the triangles or quadrilaterals in ćn.

We can now state the main result of this section.

THEOREM 3.1. If the above conditions are satisfied, then (2.10) holds.

Let us postpone the proof of the theorem and first prove two lemmas.

Below we will denote by $\Pi_{h}$ the $L^{2}$-projection from $P_{h}$ onto the space

$$
\left.Q_{h}=\left\{\mu \in L_{0}^{2}(\Omega) \mid \mu_{1 M} \text { is constant } \forall M \in:\right) \Re_{h}\right\} \text {. }
$$

Lemma 3.2. Suppose that the conditions (3.6) and (3.7) are valid. Then there is a constant $C_{1}>0$ such that for every $p \in P_{h}$ there is a $v \in V_{h}$ satisfying

$$
(\operatorname{div} v, p)=\left(\operatorname{div} v \cdot\left(I-\mathrm{II}_{h}\right) p\right) \geqslant C_{1}\left\|\left(I-\mathrm{II}_{h}\right) p\right\|_{0}^{2}
$$

and

$$
|v|_{1} \leqslant\left\|\left(I-\Pi_{h}\right) p\right\|_{0} .
$$

Proof. For every $p \in P_{h}$ we have

$$
\left(I-\Pi_{h}\right) p \in P_{0, M} \quad \forall M \in \Re_{h} \text {. }
$$

Since every $M \in: \Re_{h}$ belongs to some of the classes $\hat{E}_{\hat{M}_{i}}, i=1,2 \ldots, n$. Lemma 3.1 implies that for every $M$ there exists $v_{M} \in V_{0 . M}$ such that

$$
\left(\operatorname{div} v_{M} \cdot\left(I-\Pi_{h}\right) p\right)_{M} \geqslant C_{1}\left\|\left(I-\Pi_{h}\right) p\right\|_{0, M}^{2}
$$

and

$$
\left|c_{M}\right|_{1, M} \leqslant\left\|\left(I-\Pi_{h}\right) p\right\|_{O, M} .
$$

where $C_{1}=\min \left\langle\beta_{\hat{H}_{1}}, i=1, \ldots, n\right\}$ and the positive constants $\beta_{\hat{M}_{1}}$ are as in Lemma 3.1. Let us now define $v$ through

$$
v_{\mid M}=v_{M} \quad \forall M \in \Re_{h} .
$$

Since $v=0$ on $\partial M$ for every $M \in \mathbb{R}_{h}$ we conclude that $v \in V_{h}$ and

$$
\left(\operatorname{div} v, \Pi_{h} p\right)=0 \quad \forall p \in P_{h},
$$

and the assertion of the lemma now follows from (3.10) through (3.12).

LEMma 3.3. Suppose that the condition (3.8) is valid. Then there is a constant $C_{2}>0$ such that for every $p \in P_{h}$ there is a $g \in V_{h}$ satisfying

$$
\left(\operatorname{div} g, \Pi_{h} p\right)=\left\|\Pi_{h} p\right\|_{0}^{2} \text { and }|g|_{1} \leqslant C_{2}\left\|\Pi_{h} p\right\|_{0} .
$$

Proof. Let $p \in P_{h}$ be arbitrary. Since $\Pi_{h} p \in L_{0}^{2}(\Omega 2)$, there exists (cf. [11]) $z \in\left[H_{0}^{\prime}(\Omega)\right]^{2}$ such that

$$
\operatorname{div} z=\Pi_{h} p
$$


and

$$
|z|_{1} \leqslant C\left\|\Pi_{h} p\right\|_{0} .
$$

We will now combine some ideas from [8] and [9] in order to construct an operator $I_{h}:\left[H_{0}^{\prime}(\Omega)\right]^{2} \rightarrow V_{h}$ such that

$$
\left(\operatorname{div} I_{h} z, \mu\right)=(\operatorname{div} z, \mu) \quad \forall \mu \in Q_{h}
$$

and

$$
\left|I_{h} z\right|_{1} \leqslant C|z|_{1} .
$$

The assertion then follows from (3.13) through (3.16).

In order to define $I_{h}$ we introduce some additional notation. As the degrees of freedom of a $v \in V_{h}$ we choose the values $v_{i}=v\left(x^{i}\right), i=1,2, \ldots, q$, at the Lagrange nodes $x^{i}, i=1,2, \ldots, q$ (cf. [6], [7]). Let $w_{i}, i=1,2, \ldots, q$, be the corresponding basis functions defined by $w_{i}\left(x^{\prime}\right)=\delta_{i j}$. The support of the basis function $w_{i}$ will be denoted by $S_{i}$, and $\left|S_{i}\right|$ will stand for the area of $S_{i}$. The inter-element boundaries of the macroelements in $\mathfrak{M}_{h}$ will be denoted by $T_{i}, i=1, \ldots, \kappa$ (i.e. each $T_{1}$ is the common part of the boundaries of two neighboring macroelements). We will assume that $\mathfrak{M}_{h}$ consists of at least two macroelements so that $1 \leqslant \kappa<q$.

Due to the assumption (3.8) we may assume that for $i=1, \ldots, \kappa$ the node $x^{i} \in T_{\text {, }}$ and that supp $w_{i} \subset M_{i_{1}} \cup M_{i_{2}}$, where $M_{i_{1}}$ and $M_{i_{2}}$ are the macroelements in $\mathscr{R}_{h}$ such that $T_{i}=M_{i_{1}} \cap M_{i_{2}}$ (when $k \geqslant 2$ in (2.8) $x^{i}, i=1, \ldots, \kappa$, is taken as one of the interior nodes on an edge, of a triangle or quadrilateral, common to $M_{i_{1}}$ and $M_{i_{2}}$ ). Since $\int_{T_{1}} w_{i} d s \neq 0$, we can uniquely define $I_{h} z$ by requiring

$$
\left(I_{h} z\right)\left(x^{l}\right)=\frac{\int_{S_{l}} z d x}{\left|S_{l}\right|} \quad \text { for } i=\kappa+1, \ldots, m,
$$

and

$$
\int_{T_{1}} I_{h} z d s=\int_{T_{1}} z d s \text { for } i=1, \ldots, \kappa .
$$

Since $Q_{h}$ consists of functions that are constant on each $M \in \mathcal{O} \Re_{h}$, an integration by parts shows that condition (ii) implies (3.15). The estimate (3.16) is easily proved using a scaling argument.

The lemma is thus proved.

We close this section by giving the

Proof of Theorem 3.1. Let $p \in P_{h}$ be arbitrary, and let $v \in V_{h}, g \in V_{h}, C_{1}$ and $C_{2}$ be as in Lemma 3.2 and Lemma 3.3. Set $z=v+\delta g$, where $\delta=2 C_{1}\left(1+C_{2}^{2}\right)^{-1}$. Then we have

$$
\begin{aligned}
(\operatorname{div} z, p) & =(\operatorname{div} v, p)+\delta(\operatorname{div} g, p) \\
& \geqslant C_{1}\left\|\left(I-\Pi_{h}\right) p\right\|_{0}^{2}+\delta\left(\operatorname{div} g, \Pi_{h} p\right)+\delta\left(\operatorname{div} g,\left(I-\Pi_{h}\right) p\right) \\
& \geqslant C_{1}\left\|\left(I-\Pi_{h}\right) p\right\|_{0}^{2}+\delta\left\|\Pi_{h} p\right\|_{0}^{2}-\delta|g|_{1}\left\|\left(I-\Pi_{h}\right) p\right\|_{0} \\
& \geqslant\left(C_{1}-\frac{\delta C_{2}^{2}}{2}\right)\left\|\left(I-\Pi_{h}\right) p\right\|_{0}^{2}+\frac{\delta}{2}\left\|\Pi_{h} p\right\|_{0}^{2} \\
& =C_{1}\left(1+C_{2}^{2}\right)^{-1}\|p\|_{0}^{2}
\end{aligned}
$$


and

$$
|z|_{1} \leqslant\left\|\left(I-\mathrm{II}_{h}\right) p\right\|_{0}+\delta C_{2}\left\|\mathrm{II}_{h} p\right\|_{0} \leqslant C\|p\|_{0} .
$$

The inequalities (3.17) and (3.18) are just an alternative way of stating the condition (2.10), and the theorem is thus proved.

4. Applications. In this section we apply the theory developed in Section 3 to some mixed methods. Let us first note that all the conforming methods discussed in [4], [9] and [18] can also be analyzed using the technique of Section 3. In fact, the essence of the analysis of [9], [18] consists of verifying the condition (3.6) for macroelements consisting of only one element. Using the present technique, we obtain optimal convergence rates for both the velocity and the pressure in the examples studied in [4], [9] and [18]. Thus, our analysis shows that the assumption of [4], [9] and [18] that the mesh is quasiuniform (i.e. $h_{K} \geqslant C h$ for every $K \in \mathcal{E}_{h}$ ) can be dropped and that the suboptimal estimates for the pressure proved in [4] can be improved to optimal ones. Improvements of some of the results of [4] are also obtained in [23], but still under the quasiuniformity assumption.

The simplest method of approximation would be a piecewise linear or bilinear approximation for the velocities and a piecewise constant approximation for the pressure. It is, however, well known (cf. [15], [21]) that the corresponding mixed method in general does not satisfy (2.10). In particular, when the region $\Omega$ is rectangular and $e_{h}$ consists of rectangular elements it is well known (cf. [15]. [21]...) that there is a nonconstant. "checkerboard" function $\mu \in P_{h}$ such that (div $v, \mu)=0$ for every $v \in V_{h}$. In our first example below we propose an alternative of this method, using bilinear quadrilateral approximations for the velocities and a piecewise constant approximation for the pressure, which satisfies the stability inequality (2.10).

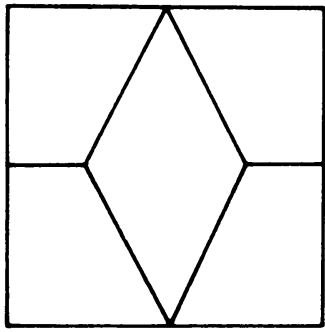

M

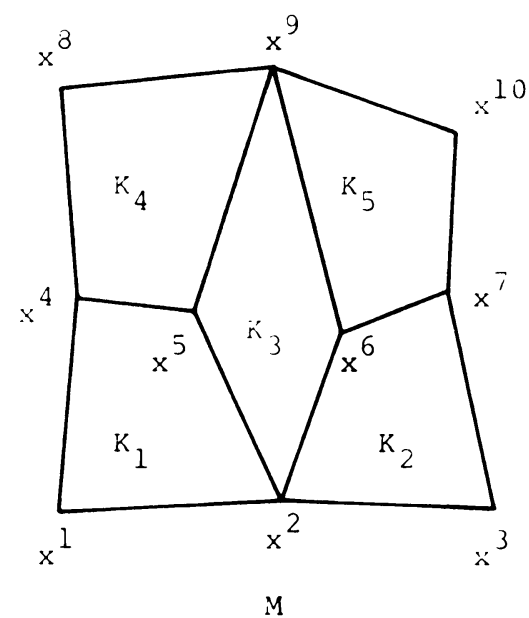

iM

Figure 1

Example 1. Consider the reference macroelement $\hat{M}$ and an arbitrary $M \in \varepsilon_{\hat{M}}$ as shown in Figure 1. Define the spaces $V_{0, M}$ and $P_{M}$ as

$$
V_{0 . M}=\left\{v \in\left[H_{0}^{1}(M)\right]^{2} \mid v_{\| \mid K} \in Q_{1}(K), i=1,2, \forall K \subset M\right\},
$$


and

$$
P_{M}=\left\{p \in L^{2}(M) \mid p_{\mid K} \text { is constant } \forall K \subset M\right\} .
$$

Let us now check the condition (3.6). Choose $v \in V_{0, M}$ such that $v\left(x^{i}\right)=0$, $i=1,2, \ldots, 10, i \neq 5$, and $v_{1}\left(x^{5}\right)=1, v_{2}\left(x^{5}\right)=0$, respectively, $v_{1}\left(x^{5}\right)=0, v_{2}\left(x^{5}\right)$ $=1$. The condition $(\operatorname{div} v, p)_{M}=0$ then gives the equations

$$
\left\{\begin{array}{l}
p_{1}\left(x_{1}^{2}-x_{1}^{4}\right)+p_{3}\left(x_{1}^{9}-x_{1}^{2}\right)+p_{4}\left(x_{1}^{4}-x_{1}^{9}\right)=0 \\
p_{1}\left(x_{2}^{2}-x_{2}^{4}\right)+p_{3}\left(x_{2}^{9}-x_{2}^{2}\right)+p_{4}\left(x_{2}^{4}-x_{2}^{9}\right)=0
\end{array}\right.
$$

where we have written $x^{i}=\left(x_{1}^{i}, x_{2}^{i}\right), i=1,2, \ldots, 10$, and $p_{i}=p_{\mid K}, i=1,2, \ldots, 5$. The equations are easily seen to be linearly independent with the only solution $p_{1}=p_{3}=p_{4}$. In the same way we conclude that the condition $(\operatorname{div} v, p)_{M}=0$, where $v$ is chosen such that $v\left(x^{i}\right)=0, i=1,2, \ldots, 10, i \neq 6$, and $v_{1}\left(x^{6}\right)=1$, $v_{2}\left(x^{6}\right)=0$, respectively, $v_{1}\left(x^{6}\right)=0, v_{2}\left(x^{6}\right)=1$, implies that $p_{2}=p_{3}=p_{5}$. The condition (3.6) is thus satisfied. Let us now define

$$
V_{h}=\left\{v \in\left[H_{0}^{1}(\Omega)\right]^{2} \mid v_{i \mid K} \in Q_{1}(K) \forall K \in C_{h}\right\}
$$

and

$$
P_{h}=\left\{p \in L_{0}^{2}(\Omega) \mid P_{\mid K} \text { is constant } \forall K \in \mathcal{C}_{h}\right\}
$$

Suppose that for every $h$ there is a macroelement partitioning $\mathfrak{N}_{h}$ such that every $M \in \mathfrak{M}_{h}$ belongs to $\mathcal{E}_{\hat{M}}$ where $\hat{M}$ is as in Figure 1. Since the conditions (3.6) and (3.8) are satisfied, Theorem 3.1 shows that the stability inequality $(2.10)$ is valid. Suppose $\Omega$ is convex. We then have $u \in\left[H^{2}(\Omega)\right]^{2}$ and $p \in H^{1}(\Omega)$ if $f \in\left[L^{2}(\Omega)\right]^{2}$ (cf. [11]), and Theorem 2.1 implies the estimates

$$
\left\|u-u_{h}\right\|_{1}+\left\|p-p_{h}\right\|_{0} \leqslant C h\left(\|u\|_{2}+\|p\|_{1}\right)
$$

and

$$
\left\|u-u_{h}\right\|_{0} \leqslant C h^{2}\left(\|u\|_{2}+\|p\|_{1}\right) \text {. }
$$

Remark. The method proposed by Le Tallec [16] can also be analyzed with the present technique, and the estimates (4.1) and (4.2) hold also for this method.

In the following examples we consider three mixed methods for which we have not found detailed error analysis in the literature.

Example 2. The Hood-Taylor method [12]. In this method the elements $K \in \bigodot_{h}$ are quadrilaterals and the approximating spaces are defined as

$$
V_{h}=\left\{v \in\left[H_{0}^{1}(\Omega)\right]^{2} \mid v_{i \mid K} \in Q_{2}(K), i=1,2, \forall K \in \bigodot_{h}\right\}
$$

and

$$
P_{h}=\left\{p \in L_{0}^{2}(\Omega) \cap C(\bar{\Omega}) \mid p_{\mid K} \in Q_{1}(K) \forall K \in \mathcal{C}_{h}\right\}
$$

The method has previously been analyzed in [4] in the case of rectangular elements. We will now derive error estimates for the general quadrilateral case. 


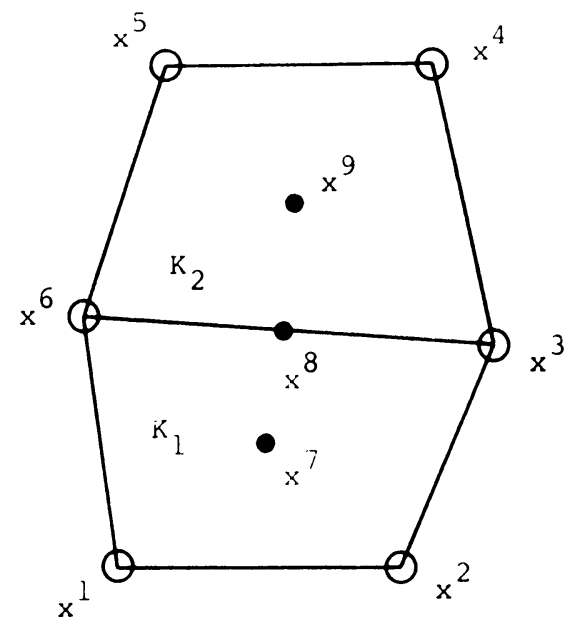

FIGURE 2

Degrees of freedom for $V_{0, M}(\bullet)$ and $P_{M}(O)$

To check the validity of the condition (3.6), let $M=K_{1} \cup K_{2}$ be a macroelement consisting of two neighboring quadrilaterals (see Figure 2). The degrees of freedom for $P_{M}$ are the values $p_{t}=p\left(x^{i}\right)$ at the nodes $x^{i}, i=1,2 \ldots 6$, and for $V_{0, M}$ they are the values $v\left(x^{\prime}\right)$ at $x^{\prime}, i=7,8,9$. Let $\hat{M}=\hat{K}_{1} \cup \hat{K}_{2}$ be the reference macroelement and assume that $\hat{K}_{1}$ and $\hat{K}_{2}$ are squares. Using the Green's formula we have

$$
(\operatorname{div} v, p)_{M}=-(v, \nabla p)_{M}=-\sum_{i=1}^{2}(v, \nabla p)_{K_{i}},
$$

for $v \in V_{0, M}$ and $p \in P_{M}$. Let $F_{M}$ be the piecewise bilinear mapping from $\hat{M}$ to $M$ and define. for $v \in V_{0 . M}$ and $p \in P_{M}, \hat{v}$ and $\hat{p}$ in $\hat{M}$ through $\hat{v}(\hat{x})=v\left(F_{M}(\hat{x})\right)$ and $\hat{p}(\hat{x})=p\left(F_{M}(\hat{x})\right)$. We can then write

$$
(v \cdot \nabla p)_{\kappa_{i}}=\int_{\hat{K}_{i}} \hat{v}(\hat{x}) J_{F_{M}}^{-T} \nabla \hat{p}(\hat{x})\left|J_{F_{M}}\right| d \hat{x}, \quad j=1,2
$$

where $J_{F_{M}}$ is the Jacobian matrix of $F_{M},\left|J_{F_{M}}\right|$ is the determinant of $J_{F_{M}}$ and $J_{F_{M}}^{-T}$ is the transpose of $J_{F_{M}}^{-1}$. Now, an inspection shows that

$$
\hat{v}(\hat{x}) J_{F_{M}}^{-T} \nabla \hat{p}(\hat{x})\left|J_{F_{M}}\right| \in Q_{3}\left(\hat{K}_{j}\right)
$$

and the integral in (4.5) can thus be exactly evaluated using the composite Simpson's rule. Further, we have $\left|J_{F_{y}}(\hat{x})\right| \neq 0$ for every $\hat{x} \in \hat{K}_{j}$. Using these facts we conclude that the condition $(v, \nabla p)_{M}=0$, where $v \in V_{0, M}$ is chosen such that $\hat{v}\left(\hat{x}^{\prime}\right)=0$, $i=8,9$, and $\hat{v}_{1}\left(\hat{x}^{7}\right)=1, \quad \hat{v}_{2}\left(\hat{x}^{7}\right)=0$, respectively, $\hat{v}_{1}\left(\hat{x}^{7}\right)=0, \hat{v}_{2}\left(\hat{x}^{7}\right)=1 \quad\left(\hat{x}^{i}=\right.$ $\left.F_{M}^{-1}\left(x^{\prime}\right), i=7,8,9\right)$, gives the equations

$$
\left\{\begin{array}{l}
p_{3}-p_{6}+p_{2}-p_{1}=0 \\
p_{6}-p_{1}+p_{3}-p_{2}=0
\end{array}\right.
$$


In the same way we get (taking $\hat{v}\left(\hat{x}^{i}\right)=0, i=7,8$, and $\hat{v}_{1}\left(\hat{x}^{9}\right)=1, \hat{v}_{2}\left(\hat{x}^{9}\right)=0$, respectively, $\left.\hat{v}_{1}\left(\hat{x}^{9}\right)=0, \hat{v}_{2}\left(\hat{x}^{9}\right)=1\right)$

$$
\left\{\begin{array}{l}
p_{4}-p_{5}+p_{3}-p_{6}=0 \\
p_{5}-p_{6}+p_{4}-p_{3}=0
\end{array}\right.
$$

The equations in (4.6) and (4.7) are linearly independent with the solution

$$
\left\{\begin{array}{l}
p_{1}=p_{3}=p_{5}=a \\
p_{2}=p_{4}=p_{6}=b
\end{array}\right.
$$

where $a$ and $b$ are arbitrary real constants. Now, choose $v \in V_{0, M}$ such that $\hat{v}\left(\hat{x}^{\prime}\right)=0, i=7,9$, and $\hat{v}_{1}\left(\hat{x}^{8}\right)=1, \hat{v}_{2}\left(\hat{x}^{8}\right)=0$, respectively, $\hat{v}_{1}\left(\hat{x}^{8}\right)=0, \hat{v}_{2}\left(\hat{x}^{8}\right)=1$. If $p \in P_{M}$ satisfies (4.8), then the condition $(v, \nabla p)_{M}=0$ gives the equations

$$
\left\{\begin{array}{l}
(a-b)\left(x_{1}^{5}+x_{1}^{4}-x_{1}^{1}-x_{1}^{2}\right)=0, \\
(a-b)\left(x_{2}^{5}+x_{2}^{4}-x_{2}^{1}-x_{2}^{2}\right)=0 .
\end{array}\right.
$$

Now, we cannot simultaneously have $x_{1}^{5}+x_{1}^{4}-x_{1}^{1}-x_{1}^{2}=0$ and $x_{2}^{5}+x_{2}^{4}-x_{2}^{1}-$ $x_{2}^{2}=0$, since it would imply that the midpoint of the side $x^{4}-x^{5}$ coincides with the midpoint of the side $x^{1}-x^{2}$. Therefore we conclude that $a=b$ and (3.6) is thus valid for $M$. In the same way one can show that (3.6) is also satisfied for a macroelement consisting of more than two quadrilaterals. The quadrilaterals in $e_{h}$ can always be grouped together to macroelements consisting of two or three quadrilaterals. There is only a finite number of different classes of such macroelements and (3.7) is thus satisfied. Theorem 3.1 and Theorem 2.1 then imply the estimates

$$
\left|u-u_{h}\right|_{1}+\left\|p-p_{h}\right\|_{0} \leqslant C h^{2}\left(\|u\|_{3}+\|p\|_{2}\right)
$$

and

$$
\left\|u-u_{h}\right\|_{0} \leqslant C h^{3}\left(\|u\|_{3}+\|p\|_{2}\right),
$$

provided that $u \in\left[H^{3}(\Omega)\right]^{2}, p \in H^{2}(\Omega)$ and $\Omega$ is convex.

Remark. If the boundary of $\Omega$ is curved, then the velocities are usually (cf. [12]) approximated with isoparametric biquadratic elements whereas the pressure is approximated with "superparametric" bilinear elements, i.e., $Q_{i}(K), i=1,2$, in (4.3), (4.4) are defined as

$$
Q_{i}(K)=\left\{\hat{p}_{i} \circ F_{K}^{-1} \mid \hat{p}_{i} \in Q_{i}(\hat{K})\right\},
$$

where $Q_{i}(\hat{K})$ is defined in (2.6) and $F_{K}: \hat{K} \rightarrow K$ is a regular biquadratic mapping as defined in [7]. For each $K \in \mathcal{C}_{h}$ let $a_{i, K}, i=1,2, \ldots, 9$, be the usual Lagrange nodes such that $a_{i, K}, i=1,2,3,4$, are the vertices of $K$. Let $\tilde{a}_{i, \tilde{K}}, i=1,2, \ldots, 9$, be the nodes for the corresponding straightsided quadrilateral $\tilde{K}$ with $\tilde{a}_{i, \tilde{K}}=a_{i, K}$ for $i=1,2,3,4$. One can now easily show that the stability inequality $(2.10)$ still holds if we have

$$
\left\|a_{i, K}-\tilde{a}_{i, \tilde{K}}\right\| \leqslant C h_{K}, \quad i=5,6, \ldots, 9, \forall K \in \mathcal{C}_{h},
$$

where $C$ stands for a sufficiently small positive constant. In the definition of the regular mapping $F_{K}$ one has the condition $\left\|a_{i, K}-\tilde{a}_{i, \tilde{K}}\right\|=O\left(h_{K}^{2}\right), i=5,6, \ldots, 9$, 
and (4.12) thus holds provided that the mesh parameter $h$ is sufficiently small. Since the approximation properties of $V_{h}$ and $P_{h}$ are as in Lemmas 2.1 and 2.2, Theorem 2.1 holds. The estimates (4.10) and (4.11) are thus also valid for the general isoparametric Hood-Taylor method.

Example 3. In this example we treat a modifaction of the previous Hood-Taylor method (cf. [14]). We assume that $\Omega$ is a rectangle (or the union of rectangles) and that the elements $K \in E_{h}$ are rectangles. The space $P_{h}$ is defined as in (4.4) and $V_{h}$ as

$$
V_{h}=\left\{\left.v \in\left[H_{0}^{\prime}(\Omega)\right]^{2}\right|_{v_{1 \mid K}} \in Q_{2}^{\prime}(K), i=1,2, \forall K \in \mathcal{L}_{h}^{\prime}\right\} .
$$

where $Q_{2}^{\prime}(K)$ is the reduced space of biquadratic polynomials defined in [6, p. 63].

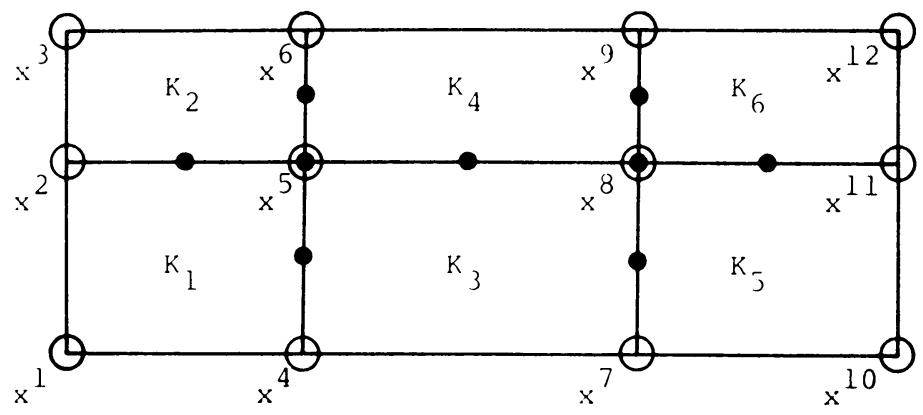

FIGURE 3

Degrees of freedom for $V_{0, M}(\bullet)$ and for $P_{M}(0)$

Let us now check the validity of the crucial condition (3.6). Consider a macroelement $M$ consisting of six rectangles arranged as in Figure 3. Consider first the macroelement $M_{1}=\cup_{1=1}^{4} K_{1}$. The condition ( $\left.\operatorname{div} v, p\right)_{M_{1}}=0$, for every $v \in V_{0, M_{1}}$. gives a system of ten equations for the nine pressures $p_{1}=p\left(x^{i}\right), i=1,2, \ldots, 9$. The system (which we omit to write out explicitly) is easily seen to have a rank of seven and the nontrivial solution

$$
\left\{\begin{array}{l}
p_{1}=p_{3}=p_{9}=p_{7}=a, \\
p_{2}=p_{4}=p_{6}=p_{8}=b, \\
p_{5}=\frac{1}{2}(a+b),
\end{array}\right.
$$

where $a$ and $b$ are arbitrary real constants. Repeating this argument for the macroelement $M_{2}=\cup_{i=3}^{6} K_{i}$, we conclude similarly that

$$
\left\{\begin{array}{l}
p_{4}=p_{6}=p_{10}=p_{12}=c, \\
p_{5}=p_{7}=p_{9}=p_{11}=d, \\
p_{8}=\frac{1}{2}(c+d) .
\end{array}\right.
$$

Now, if $p$ satisfies $(\operatorname{div} v, p)_{M}=0$ for every $v \in V_{0, M}$, then it has to satisfy both (4.13) and (4.14), which is possible only if $a=b=c=d$, i.e. $p$ is a constant in $M$. The condition (3.6) is thus satisfied. In the same way we conclude that if a macroelement contains another macroelement which is equivalent to the macroelement in Figure 3, then (3.6) is satisfied. There is now a finite number of classes of macroelements, consisting of less than or equal to 24 rectangles, which satisfies (3.6). 
Since for each $h$ there is an $\Re_{h}$ where each $M \in \mathfrak{M}_{h}$ belongs to one of the above classes, Theorem 3.1 holds and the error estimates (4.10) and (4.11) are valid.

Example 4. In this method, which is being increasingly used in practice (cf. [10]), the space $V_{h}$ is defined as in (4.3), whereas one uses a discontinuous approximation for the pressure,

$$
P_{h}=\left\{p \in L_{0}^{2}(\Omega) \mid p_{\mid K} \in P_{1}(K) \forall K \in \mathcal{C}_{h}\right\} .
$$

Let us now show that the condition (3.6) is valid for macroelements consisting of only one quadrilateral. On an arbitrary quadrilateral $K \in \mathcal{C}_{h}, p \in P_{h}$ can be written as

$$
p_{\mid K}=a_{0, K}+a_{1, K} x_{1}+a_{2, K} x_{2} .
$$

Let $x^{0}$ be the interior node in $K$, and let $w_{0}$ be the corresponding basis function of $V_{h}$. Choose $v \in V_{0, K}$ such that $v_{1}\left(x^{0}\right)=1$ and $v_{2}\left(x^{0}\right)=0$. We then obtain

$$
(\operatorname{div} v, p)_{K}=-(v, \nabla p)_{K}=-a_{1, K} \int_{K} w_{0} d x
$$

Since $\int_{K} w_{0} d x>0$, the condition $(\operatorname{div} v, p)_{K}=0$ implies that $a_{1, K}=0$. In the same way, choosing $v \in V_{0, K}$ such that $v_{1}\left(x^{0}\right)=0$ and $v_{2}\left(x^{0}\right)=1$, we conclude that the condition ( $\operatorname{div} v, p)_{K}=0$ gives $a_{2, K}=0$. The condition (3.6) is thus satisfied for an arbitrary $M=K \in \mathcal{C}_{h}$. We may then choose $\mathfrak{T}_{h}=\mathcal{C}_{h}$ in (3.7) and (3.8), and so we, once again, obtain the estimates (4.10) and (4.11).

Remarks. (1) As in the remark following Example 2 we can conclude that the stated error estimates remain valid for the general isoparametric method.

(2) Of the methods treated in Examples 2, 3 and 4 the last one seems superior, due to the fact that the discrete system can in this case be solved effectively using the penalty method, cf. [2], [10], [17].

(3) A method which is also often used in practice (cf. [3], [13], [17]) consists of the following choices for $V_{h}$ and $P_{h}$ :

$$
\begin{aligned}
& V_{h}=\left\{v \in\left[H_{0}^{1}(\Omega)\right]^{2} \mid v_{i \mid K} \in Q_{2}(K), i=1,2, \forall K \in \mathcal{C}_{h}\right\}, \\
& P_{h}=\left\{p \in L_{0}^{2}(\Omega) \mid p_{\mid K} \in Q_{1}(K) \forall K \in \mathcal{C}_{h}\right\} .
\end{aligned}
$$

The method has originally been introduced in the engineering literature as a penalty method with "reduced selective integration", cf. [3], [13], [17].

The method does not satisfy the condition (3.6), so we cannot apply the theory developed in this paper. For rectangular elements it is, however, possible to analyze the method using the technique developed in [15]. The error estimates one obtains in this way are [22]

$$
\begin{aligned}
\left|u-u_{h}\right|_{1} & \leqslant C h^{2}\left(|u|_{3}+|u|_{4, q}+|p|_{2}\right), \\
\left\|u-u_{h}\right\|_{0} & \leqslant C h^{3}\left(|u|_{3}+|u|_{4, q}+|p|_{2}\right)
\end{aligned}
$$

and

$$
\left\|p-p_{h}\right\|_{0} \leqslant C h\left(|u|_{3}+|u|_{4, q}+|p|_{2}\right)
$$


where $q>1$ and $|\cdot|_{4 . q}$ stands for the usual seminorm in the Sobolev space $W^{4.4}(\Omega)$. From the estimates one sees that the pressure does not converge with the optimal rate, a fact also observed in practical computations [21]. In [21] it is also noted that one can get a good approximation for the pressure by simply omitting the $x_{1} x_{2}$-component in each element in the computed $p_{h}$, and this can also be proved theoretically [22]. The resulting smoothed pressure then converges with the optimal $O\left(h^{2}\right)$-rate. In view of this analysis, the role of the $x_{1} x_{2}$-component is mainly disturbing and it is therefore natural to drop it from $P_{h \mid K}$. This leads back to (4.10).

Acknowledgement. The author is grateful to Professor Juhani Pitkäranta for suggesting this study and for numerous fruitful discussions during the course of the work.

Institute of Mathematics

Helsinki University of Technology

SF-02150 Espoo 15. Finland

1. I. BabušKa. "The finite element method with Lagrangian multipliers," Numer. Math., v. $20,1973$. pp. 179-192.

2. M. Bercovier. "Perturbation of mixed variational problems. Application to mixed finite element methods". RAIRO Anal. Numer., v. 12, 1978, pp. 211-236.

3. M. Bercovier \& M. Engelman, "A finite element method for the numerical solution of viscous incompressible flows". J. Comput. Phi's., v. 30, 1979, pp. 181-201.

4. M. Bercovier \& O. Pironneau, "Error estimates for finite element solution of the Stokes problem in the primitive variables," Numer. Math., v. 33, 1979. pp. 211-224.

5. F. Brezzi, "On the existence, uniqueness and approximation of saddle-point problems arising from Lagrangian multipliers," RAIRO Ser. Rouge, v. 8. 1974, pp. 129-151.

6. P. G. Ciarlet, The Finite Element Method for Elliptic Problems. North-Holland, Amsterdam. 1978.

7. P. G. Ciarlet \& P. A. Raviart, "Interpolation theory over curved elements, with applications to finite element methods," Comput. Methods Appl. Mech. Engrg.. v. I. 1972. pp. 217-249.

8. P. Clemént. "Approximation by finite elements using local regularization," RAIRO Ser. Rouge,v. 9. 1975, pp. 77-84.

9. M. Crouzeix \& P. A. Raviart, "Conforming and nonconforming finite element methods for solving the stationary Stokes equations," RAIRO Ser. Rouge, v. 7, 1973, pp. 33-76.

10. M. Engelman, R. Sani, P. Gresho \& M. Bercovier, "Consistent vs. reduced integration penalty methods for incompressible media using several old and new elements," Internat. J. Numer. Methods Fluids, v. 2. 1982, pp. 25-42.

11. V. Girault \& P. A. Raviart, Finite Element Approximation of the Navier-Stokes Equations, Lecture Notes in Math., Vol. 749. Springer. Berlin. 1979.

12. P. HOOD \& C. TAYLOR, "Navier-Stokes equations using mixed interpolation," Finite Element Methods in Flow Problems (J. T. Oden. ed.), UAH Press, Huntsville, Alabama, 1974, pp. 121-131.

13. T. J. Hughes, W. K. LIU \& A. BROOKS, "Finite element analysis of incompressible viscous flows by the penalty function formulation," J. Comput. Phys., v. 30, 1979, pp. 1-60.

14. P. Huyakorn, C. TAylor, R. Lee \& P. Gresho, “A comparison of various mixed-interpolation finite elements for the Navier-Stokes equations," Comput. \& Fluids, v. 6. 1978, pp. 25-35.

15. C. Johnson \& J. PitKäranta, "Analysis of some mixed finite element methods related to reduced integration," Math. Comp., v. 38, 1982, pp. 375-400.

16. P. Le TAllec, "Compatibility condition and existence results in discrete finite incompressible elasticity." Comput. Methods Appl. Mech. Engrg., v. 27. 1981. pp. 239-259.

17. D. Malkus \& T. J. Hughes, "Mixed finite element methods-reduced and selective integration techniques: a unification of concepts," Comput. Mehtods Appl. Mech. Engrg., v. 15, 1978, pp. 63-81.

18. L. MANSFIELD, "Finite element subspaces with optimal rates of convergence for the stationary Stokes problem." RAIRO Anal. Numer., v. 16, 1982, pp. 49-66.

19. J. Pitkäranta, "On a mixed finite element method for the Stokes problem in $R^{3}, R A I R O$ Anal. Numer., v. 16, 1982, pp. 275-291. 
20. J. Pitkäranta \& R. Stenberg, “Analysis of some mixed finite element methods for plane elasticity equations," Math. Comp., v. 41, 1983, pp. 399-423.

21. R. Sani, P. Gresho, R. Lee \& D. Griffiths, "The cause and cure (?) of the spurious pressures generated by certain GFEM solutions of the incompressible Navier-Stokes equations," Internat. J. Numer. Methods Fluids, v. 1, 1981, Part 1, pp. 17-44; Part 2, pp. 171-204.

22. R. Stenberg, Mixed Finite Element Methods for Two Problems in Elasticity Theory and Fluid Mechanics, Licentiate thesis, Helsinki University of Technology, 1981.

23. R. VerfürT, Error Estimates for a Mixed Finite Element Approximation of the Stokes Equations, Ruhr-Universität Bochum, 1982. (Preprint.) 